\title{
Triiodothyronine Represses MUC5AC Expression by Antagonizing Sp1 Binding to Its Promoter in Human Bronchial Epithelial HBE16 Cells
}

\author{
Xiaolong Wang, ${ }^{1}$ Qi Li, ${ }^{1}$ Xiangdong Zhou, ${ }^{1}$ Victor P. Kolosov, ${ }^{2}$ and Juliy M. Perelman ${ }^{2}$ \\ ${ }^{1}$ Division of Respiratory Medicine, Second Affiliated Hospital, Chongqing Medical University, Chongqing 400010, China \\ ${ }^{2}$ Far Eastern Scientific Center of Physiology and Pathology of Respiration, Siberian Branch, \\ Russian Academy of Medical Sciences, 675000 Blagoveschensk, Russia
}

Correspondence should be addressed to Xiangdong Zhou, zxd999@263.net

Received 27 August 2011; Revised 9 November 2011; Accepted 9 November 2011

Academic Editor: Anton M. Jetten

Copyright ( $) 2012$ Xiaolong Wang et al. This is an open access article distributed under the Creative Commons Attribution License, which permits unrestricted use, distribution, and reproduction in any medium, provided the original work is properly cited.

\begin{abstract}
Mucus hypersecretion is a distinguished feature of chronic inflammatory airway diseases. Interestingly, in this condition thyroid function is impaired with decreased level of triiodothyronine (T3), indicating potential link between low level of T3 and mucus hypersecretion. But the underlying mechanisms are poorly understood. In this study we aimed to elucidate the effect of T3 on MUC5AC secretion in human bronchial epithelial HBE16 cells and further investigate how T3 regulates MUC5AC gene expression at transcriptional level. By RT-PCR and ELISA we showed that T3 inhibited MUC5AC mRNA expression and protein secretion in HBE16 cells. Furthermore, luciferase assay and site-directed mutagenesis analysis demonstrated that T3 repressed MUC5AC expression at transcriptional level and the mechanism might partly lie in the specific inhibition of Sp1 binding to the promoter. Our results suggest that decreased T3 level leads to the release of repression of MUC5AC expression and thus contributes to mucus hypersecretion.
\end{abstract}

\section{Introduction}

Mucus hypersecretion is a notable aspect of a variety of chronic inflammation diseases including asthma, chronic bronchitis, bronchiectasis, and cystic fibrosis $[1,2]$. Excessive production of mucus leads to airway mucus obstruction, thus contributing to the morbidity and mortality in these diseases [3]. As the major component of mucus secretions, mucins consist of a family of high-molecular weight glycoproteins synthesized by the mucosal epithelial cells that line digestive and reproductive tracts, middle ear, and trachea [4]. To date, 20 mucin genes (MUC for human and Muc for other species) have been identified. Among them, MUC2, MUC5AC, and MUC5B are crucially implicated in the pathogenesis of respiratory diseases [5]. Under normal condition, mucins protect the epithelial cells by binding and trapping the inhaled bacteria and viruses for mucociliary clearance. However, under diseased conditions such as chronic obstructive pulmonary disease (COPD), the mucociliary clearance mechanism becomes defective, and the excessive production of mucin will lead to airway obstruction [6].

Notably, COPD is characterized by chronic inflammation not only in the pulmonary compartment, but also in systemic circulation. Several factors such as hypoxemia, exacerbation, drugs, and malnutrition may lead to endocrinological changes in COPD. In particular, alterations in thyroid function tests are common in chronic systemic diseases including chronic heart failure, chronic liver or hematologic diseases, cancer, diabetes, connective tissue diseases, and COPD [7]. Frequently detected abnormality of thyroid function in nonthyroidal illness (NTI) includes a decreased level of total triiodothyronine (TT3) as well as free triiodothyronine (fT3). Interestingly, in diseased conditions such as COPD it is frequently observed that thyroid function is impaired with a decreased level of triiodothyronine (T3), indicating potential 
link between low T3 level and mucus hypersecretion. But the underlying mechanisms are poorly understood.

Here we aim to address the effect of T3 on MUC5AC secretion using the human bronchial epithelial cells HBE16 as a model. By luciferase assay we found that T3 repressed MUC5AC expression, and we further characterized the potential transcription factors and the corresponding binding sites in MUC5AC promoter involved in this regulation.

\section{Materials and Methods}

2.1. Reagents. The human bronchial epithelial cells HBE16 (a generous gift from Department of Pathology, Chongqing Medical University) were cultured in RPMI-1640 (supplemented with $10 \%$ FBS, $100 \mathrm{U} / \mathrm{mL}$ penicillin, and $100 \mu \mathrm{g} / \mathrm{mL}$ streptomycin) at $37^{\circ} \mathrm{C}$ in a humidified atmosphere containing $95 \%$ air and 5\% $\mathrm{CO}_{2}$. The cells were seeded at a final concentration of $1 \times 10^{5}$ cells $/ \mathrm{mL}$ in serum-free medium and then treated with $5 \mathrm{nM}$ all-trans-retinoic acid (RA) to maintain the basic level of MUC5AC expression. In experimental group, additional $\mathrm{T} 3$ was added to the culture medium to final concentration of $0.1,1$, and $10 \mathrm{nM}$, respectively. After $24 \mathrm{~h}$, the cells and conditioned media were collected to determine MUC5AC level.

2.2. RT-PCR. Total RNA was isolated from HBE16 cells using Trizol reagent (Roche) according to the manufacturer's instructions, and $1 \mu \mathrm{g}$ of total RNA was used for reverse transcription in a $20 \mu \mathrm{L}$ final volume with AMV Reverse Transcriptase (Promega) according to the manufacturer's protocol. PCR was then performed on $1 \mu \mathrm{L}$ of cDNA using following primers: MUC5AC 5'-TCAACGGAGACTGCGAGTACAC- $3^{\prime}$ and $5^{\prime}$-CTTGATGGCCTTGGAGCA- $3^{\prime}$, amplicon $130 \mathrm{bp}$; GAPDH $5^{\prime}$-ACCACAGTCCATGCCATCAC$3^{\prime}$ and $5^{\prime}$-TCCACCCTGTTGCTGTA-3', amplicon $491 \mathrm{bp}$. PCR products were identified by $2 \%$ agarose gel electrophoresis, and the relative level of MUC5AC mRNA was analyzed based on the ratio to GAPDH.

2.3. MUC5AC ELISA. MUC5AC in the conditioned media was measured by ELISA. $50 \mu \mathrm{L}$ of the media was incubated with bicarbonate-carbonate buffer $(50 \mu \mathrm{L})$ at $40^{\circ} \mathrm{C}$ in a 96-well plate until dry. Plates were washed three times with PBS and blocked with 2\% BSA, fraction V (Sigma) for $1 \mathrm{~h}$ at room temperature. Plates were then washed three times with PBS and incubated with $50 \mu \mathrm{L} /$ well of mouse monoclonal MUC5AC antibody (Neomarkers, CA) at $1: 100$ dilution in PBST (PBS containing $0.05 \%$ Tween 20). After $1 \mathrm{~h}$, plates were washed three times with PBS and incubated with $100 \mu \mathrm{L} /$ well of HRP-goat anti-mouse lgG conjugate (Roche) at $1: 10,000$ dilution. After $1 \mathrm{~h}$, plates were washed three times with PBS. Color reaction was developed with horseradish peroxidase solution (Kirkegaard \& Perry Laboratories) and stopped with $1 \mathrm{M} \mathrm{H}_{2} \mathrm{SO}_{4}$. Absorbance was read at $450 \mathrm{~nm}$. The results were expressed as ratio of MUC5AC to standard product.

2.4. Construction of MUC5AC Promoter Plasmids. Different MUC5AC promoter regions were PCR-amplified from genomic DNA of HBE16 cell using the following primers: $-1300 /+48$ 5'-GTCGGTACCAATGGAGAAGTAGGCAATCATA- ${ }^{\prime}$ and $5^{\prime}$-GTACTCGAGCAACACTCATTGTGTGGACG-3'; $-689 /+48 \quad 5^{\prime}$-AGTGGTACCTGAGGGACGCCTTGGCTCACT- $3^{\prime}$ and $5^{\prime}$-GTACTCGAGCAACACTCATTGTGTGGACG-3'; $-324 /+48$ 5'-ACAGGTACCCTTAAGGCTGTTCTGACCACT- $3^{\prime}$ and $5^{\prime}$-GTACTCGAGCAACACTCATTGTGTGGACG-3'; -64/+48 5' -ACAGGTACCCGTGAAGCACGGGGCTGGA- $3^{\prime}$ and $5^{\prime}$-GTACTCGAGCAACACTCATTGTGTGGACG-3'. The PCR products were subcloned into the pGL3-basic vector using KpnI/XhoI sites to construct pGL3-MUC5AC-promoter plasmids and confirmed by sequencing.

2.4.1. Construction of Sp1 and NF- $\kappa B$ Expression Plasmids. The coding sequences for human Sp1 and NF- $\kappa$ B were PCR-amplified from human full-length cDNA using the following primers: Sp1 5' -TTCTGCCCACCCACGTGAAGCA- $3^{\prime}$ and $5^{\prime}$-CGGGGCCTGTGGTTCCCCCT- ${ }^{\prime}$; NF- $\kappa$ B $5^{\prime}$-AGGACCCCTGCAGGCCAG- $3^{\prime}$ and $5^{\prime}$-GGCCACATACCCCACCCTAGAC- $3^{\prime}$.The PCR products were subcloned into pCMV3.0b vector using Kpn I/XhoI sites to construct $\mathrm{Sp} 1$ and NF- $\kappa \mathrm{B}$ expression plasmids, and then confirmed by sequencing.

2.5. Cell Transfection and Luciferase Assay. HBE16 cells were seeded in 24-well plate and cotransfected with MUC5AC promoter plasmids and pRLSV40 (internal control) using the Lipofect AMINE Plus reagent (Invitrogen, USA) following the manufacturer's protocol. T3 was added to a final concentration of $1 \mathrm{nM} 24 \mathrm{~h}$ after transfection and incubated for another $24 \mathrm{~h}$. Then cell lysate was collected, and firefly and renilla luciferase activities were measured with a TD20/20 luminometer (USA). In other cases, HBE16 cells were cotransfected with three plasmids (MUC5AC promoter plasmids, transcriptional factor expression plasmids, and pRL-SV40) and cocultured with T3 for $48 \mathrm{~h}$. Then cells were lyzed and assayed for luciferase activity. Each experiment was performed in triplicate and repeated at least four times.

2.6. Site-Directed Mutagenesis of $-324 /+48$ Region of MUC$5 A C$ Promoter. Site-directed mutagenesis of potential SP-1 binding site within MUC5AC $-324 /+48$ promoter region was performed using super high-fidelity DNA polymerase primer STARTM (Takara) with pGL3-MUC5AC -324/ +48 plasmid as template and the following primers: $5^{\prime}$-TTCTGCCCACCCACGTGAAGCA-3' and 5' -CGGGGCCTGTGGTTCCCCCT-3'.

2.7. Statistical Analysis. All data were expressed as means \pm SE. Data were analyzed by ANOVA and Dunnett's $t$ test using SPSS10.0 software. $P<0.05$ was considered as significant difference and $P<0.01$ as highly significant difference.

\section{Results}

3.1. T3 Inhibits MUC5AC mRNA Expression and Protein Secretion in HBE16 Cells. RT-PCR and ELISA analysis demonstrated that T3 inhibited MUC5AC mRNA expression 


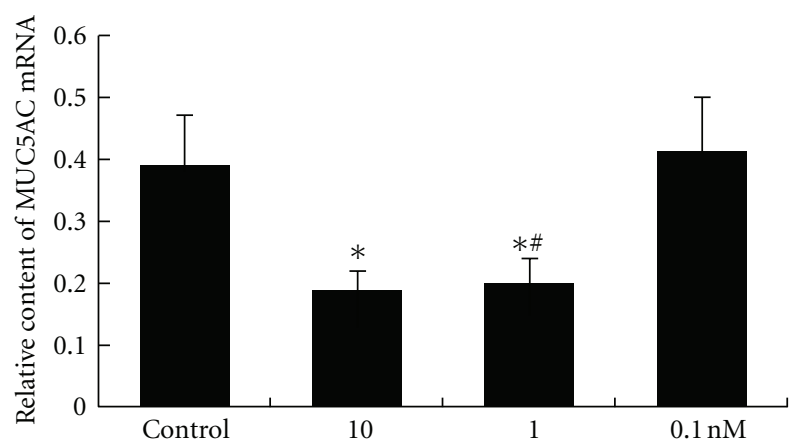

(a)

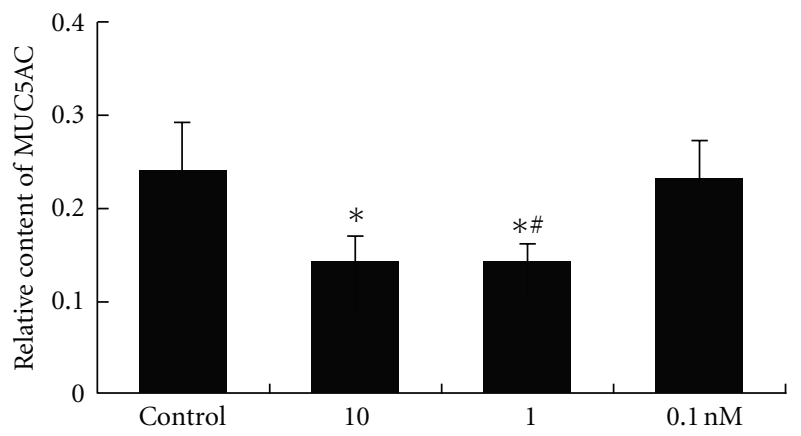

(b)

Figure 1: T3 inhibits MUC5AC mRNA expression and protein secretion in HBE16 cells. (a) MUC5AC mRNA level was determined by RT-PCR. (b) Secreted MUC5AC protein level was determined by ELISA. ${ }^{*} P<0.05$ versus control group; ${ }^{\#} P>0.05$ versus $10 \mathrm{nM}$ group.

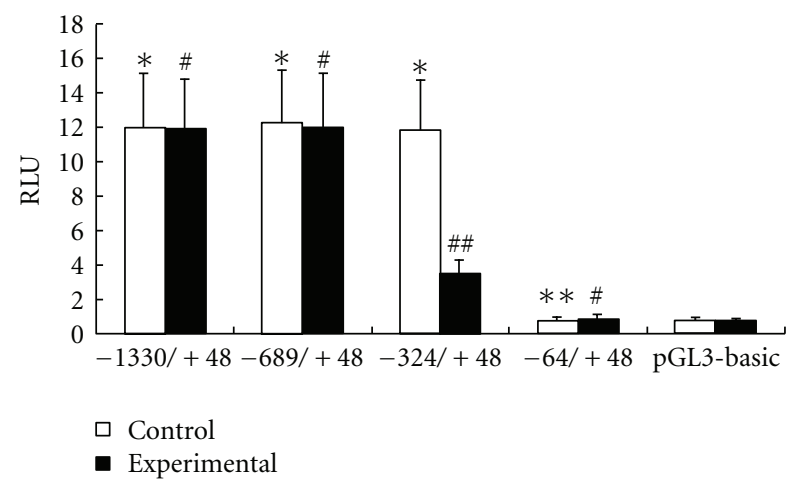

FIGURE 2: T3 inhibits the transcription of MUC5AC in HBE16 cells. The luciferase activity was determined as described in Materials and Methods. Control group: different MUC5AC promoter plasmids in the absence of T3; experimental group: different MUC5AC promoter plasmids in the presence of T3. ${ }^{*} P<0.01$ versus pGL3basic control group; ${ }^{* *} P>0.05$ versus pGL3-basic control group; ${ }^{\#} P>0.05$ versus the same promoter control group; ${ }^{\# \#} P<0.01$ versus the same promoter control group.

and protein secretion in HBE16 cells at the concentration of 1 and $10 \mathrm{nM}$ (Figure 1). However, the inhibitory effects were not in a dose-dependent manner because no statistical difference was found between $1 \mathrm{nM}$ group and $10 \mathrm{nM}$ group. Taken together, these results suggest that T3 inhibits MUC5AC expression.

\subsection{T3 Regulates the Transcription of MUC5AC in HBE16} Cells. To explore whether T3 regulates MUC5AC expression at transcriptional level, different regions of MUC5AC promoter were characterized. As shown in Figure 2, the luciferase activity was significantly upregulated in HEB16 cells transfected with MUC5AC promoter constructs containing fragment $-1,330 /+48,-689 /+48,-324 /+48(P<$ $0.01)$ but not $-64 /+48(P>0.05)$ as compared with pGL3basic vector control group, suggesting that $-324 /+48$ comprises the effective region for transcriptional regulation of MUC5AC. Notably T3 showed inhibitory effect on luciferase activity only with MUC5AC promoter construct containing fragment $-324 /+48$, thus indicating that $\mathrm{T} 3$ indeed regulates MUC5AC expression at transcriptional level.

3.3. T3 Selectively Antagonizes Sp1 but Not NF- $\kappa B$-Dependent Transcription of MUC5AC in HBE16 Cells. To further explore the potential mechanism involved in the downregulation of MUC5AC expression by T3, we assumed that T3 may inhibit the combination of transciption factors with MUC5AC promoter. To test this we made $\mathrm{Sp} 1$ and $\mathrm{NK}-\kappa \mathrm{B}$ expression plasmids and cotransfect them with MUC5AC promoter plasmids into HEB16 cells. Luciferase assay demonstrated that Sp1 upregulated the luciferase activity only in combination with $-324 /+48$ region of MUC5AC promoter, and T3 could significantly inhibit Sp1-stimulated luciferase activity (Figure 3(a)). These results suggest that Sp1 binds to $-324 /+48$ region of MUC5AC promoter to drive MUC5AC transcription and T3 may inhibit the binding of Sp1 to this region. To confirm that the inhibitory effect of T3 we observed is not artificial we also tested NF- $\kappa \mathrm{B}$ and found that similar to Sp1, NF- $\kappa$ B upregulated the luciferase activity only in combination with $-324 /+48$ region of MUC5AC promoter, suggesting that NF- $\kappa \mathrm{B}$ also binds to $-324 /+48$ region of MUC5AC promoter to drive MUC5AC transcription. However, T3 could not inhibit NF- $\kappa$ B-stimulated luciferase activity (Figure 3(b)). To provide further evidence that T3 specifically inhibits the binding of Sp 1 to $-324 /+48$ region of MUC5AC promoter, we employed site-directed mutagenesis to disrupt the potential Sp1 combined site within this region and performed luciferase assay. The results demonstrated that T3 failed to inhibit Sp1-stimulated luciferase activity in the presence of mutant MUC5AC promoter (Figure 3(c)). The putative Sp 1 binding site was located at $-80 \mathrm{bp}$ region of MUC5AC promoter. Taken together, these data demonstrate that $\mathrm{T} 3$ selectively antagonizes $\mathrm{Sp} 1$ but not NF- $\kappa \mathrm{B}$-dependent transcription of MUC5AC, indicating that T3 specifically inhibits the binding of Sp1 to MUC5AC promoter.

\section{Discussion}

T3 has been demonstrated to repress MUC5AC expression and reduce secreted mucus in normal human tracheobronchial epithelial NHTBE cells [8]. Additional studies 


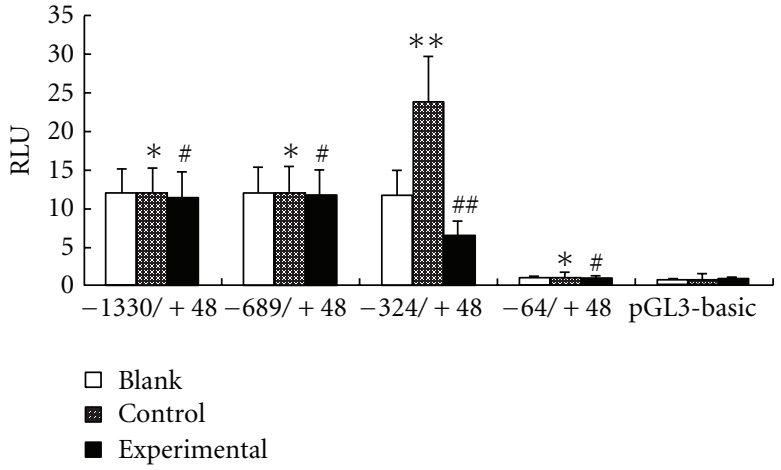

(a)

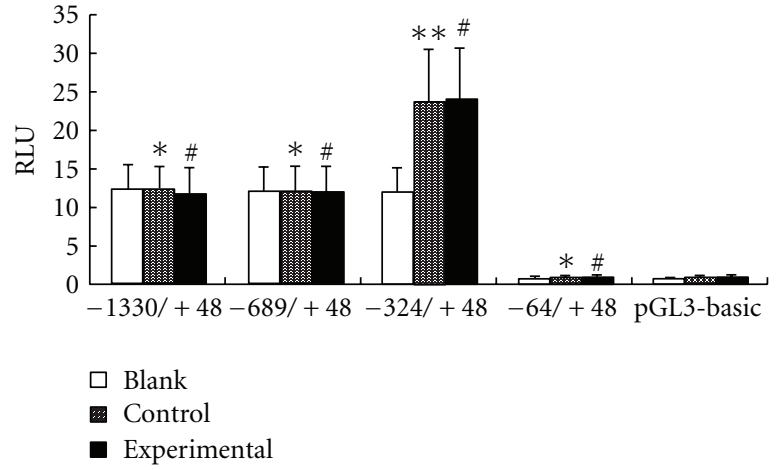

(b)

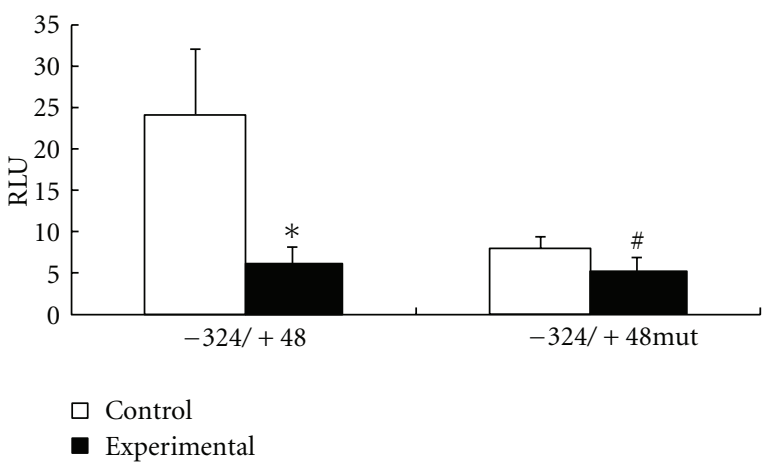

(c)

FIGURE 3: T3 selectively antagonizes Sp1 but not NF- $\kappa$ B-dependent transcription of MUC5AC in HBE16 cells. (a) After the cells were cotransfected with Sp1 expression plasmid, the luciferase activity was determined as described in Materials and Methods. Blank: different MUC5AC promoter plasmids only; control: different MUC5AC promoter plasmids plus Sp1 expression plasmid in the absence of T3; experimental: different MUC5AC promoter plasmids plus Sp1 expression plasmid in the presence of T3. ${ }^{*} P>0.05$ versus blank; ${ }^{* *} P<0.01$ versus blank; ${ }^{\#} P>0.05$ versus control; ${ }^{\# \#} P<0.01$ versus control. (b) After the cells were cotransfected with NF- $\kappa \mathrm{B}$ expression plasmid, the luciferase activity was determined as described in Materials and Methods. Blank: different MUC5AC promoter plasmids only; control: different MUC5AC promoter plasmids plus NF- $\kappa$ B expression plasmid in the absence of T3; experimental: different MUC5AC promoter plasmids plus NF- $\kappa \mathrm{B}$ expression plasmid in the presence of T3. ${ }^{*} P>0.05$ versus blank; ${ }^{* *} P<0.01$ versus blank; ${ }^{\#} P>0.05$ versus control. (c) After the cells were cotransfected with Sp1 expression plasmid and MUC $-324 /+48$ promoter plasmid or its mutation form (mut), the luciferase activity was determined as described in Materials and Methods. Control: in the absence of T3; experimental: in the presence of T3. ${ }^{*} P<0.01$ versus $-324 /+48$ control group; ${ }^{*} P>0.05$ versus $-324 /+48$ mut control group.

showed that T3 inhibits MUC5AC expression by decreasing retinoid receptor levels in human bronchial epithelial cells $[9$, 10]. Nevertheless, few studies have addressed the effect of T3 on mucin expression in the context of mucus hypersecretion. Therefore in this study we employed human bronchial epithelial HBE16 cells as the model to investigate the effect of T3 on MUC5AC expression and the underlying mechanism.

Airway epithelial cells cultured under RA-deficient condition undergo squamous differentiation, and RA treatment resulted in the restoration of the mucous phenotype and mucus hypersecretion [11]. In our study, $5 \mathrm{nM}$ all-transRA was used to maintain the basic expression of MUC5AC. As the first step to examine the influence of $\mathrm{T} 3$ on mucin expression, we conducted $\mathrm{T} 3$ concentration response studies. The results showed that as few as $1 \mathrm{nM}$ T3 significantly suppressed MUC5AC mRNA and protein expression (Figure 1). The physiological concentration of T3 has been defined as $10 \mathrm{nM}, 0.1-2.5 \mathrm{nM}$, or $1-10 \mathrm{nM}$ in previous reports, but
$1 \mathrm{nM}$ is generally accepted as a physiological concentration of T3 [12]. In this study we used T3 at $10 \mathrm{nM}$ at maximum but mainly used $\mathrm{T} 3$ at $1 \mathrm{nM}$, suggesting that our results are in a physiological setting.

The biological effects of T3 are mediated via retinoic acid receptors (RARs), retinoid X receptors (RXRs), and thyroid receptors (TRs), and both RARs and TRs require heterodimerization with RXRs for optimal transcriptional activity [13]. Although TR-RXR and RAR-RXR recognize the same core sequence of direct repeats (i.e., AGGTCA) in the promoter regions of target genes, they exhibit binding specificity as determined by the nucleotides between the direct repeats, and TR has been shown to activate or repress transcription in either a ligand-dependent or a ligandindependent manner, depending on the target genes [14]. For example, Tomic et al. reported that T3 suppressed keratin expression in epidermal keratinocytes by binding to negative response elements (nTRE) in the promoter region 
[15]. Interestingly, Moon et al. reported that RA upregulated and T3 downregulated MUC expression in cultured human middle ear epithelial cells [16]. Unfortunately, few studies have followed up to address the functional interaction between RA and T3 in the regulation of MUC expression.

To explore whether T3 suppresses MUC5AC expression in HBE16 by binding to nTRE in the promoter region of MUC5AC, we characterized MUC5AC promoter by luciferase assay and found that MUC5AC promoter activity region lies in the region of -1130 to -64 , since only promoter plasmid containing $-64 /+48$ failed to show transcription activity (Figure 2).

To further examine whether T3 represses MUC5AC expression via modulating its promoter activity, we added T3 to HBE16 cells transfected with MUC5AC promoter plasmids. The results showed that $\mathrm{T} 3$ had inhibitory effect only in cells transfected with plasmid containing $-324 /+48$ region (Figure 2). Interestingly, this region contains one proximal area of core start-CACCC, one binding site for NF- $\kappa \mathrm{B}$, and three binding sites for Sp1, and point mutation of CACCC had minor impact on the promoter activity of MUC5AC as reported previously [17]. Thus we hypothesized that NF$\kappa \mathrm{B}$ and/or Sp1 might be involved in T3-mediated repression of MUC5AC expression. Our results demonstrate that both NF- $\kappa B$ and Sp1 stimulate MUC5AC transcription by binding the $-324 /+48$ region, but only Sp1-stimulated transcription can be inhibited by T3. In addition, we disrupted the potential Sp1 combined site within the $-324 /+48$ region by site-directed mutagenesis and observed that T3 was unable to inhibit Sp1-stimulated transcription activity under this condition (Figure 3(c)). Gray et al. reported that the -1250 to -1200 region of MUC5AC promoter was crucial for T3mediated suppression of MUC5AC transcription [9]. The different mechanisms by which $\mathrm{T} 3$ mediates the suppression of MUC5AC expression may explain why the promoter region we identified is different from that by Gray et al. Their results suggest that T3 may interact with RAR and TR to modulate the -1250 to -1200 region of MUC5AC promoter, while our data indicate that T3 regulate $\mathrm{Sp}-1$ binding in $-324 /+48$ region to repress MUC5AC transcription. Significantly, our results that T3 inhibits MUC5AC expression through influencing the binding of Sp1 but not TR to MUC5AC promoter are consistent with the emerging concept that a direct binding of TR to DNA is dispensable for T3-dependent gene suppression $[18,19]$.

\section{Conclusion}

Our results suggest that decreased T3 level leads to the release of repression of MUC5AC expression and thus contributes to mucus hypersecretion. Modulation of T3 level may provide a novel therapeutic approach for chronic inflammatory airway diseases associated with mucus hypersecretion.

\section{Conflict of Interests}

The authors declare that there is no conflict of interests.

\section{Acknowledgments}

This work was supported by Grants from the National Nature Science Foundation of China (no. 30770951), and China-Russia Cooperation Research Foundation (no. 81011120108).

\section{References}

[1] H. W. J. Young, C. X. Sun, C. M. Evans, B. F. Dickey, and M. R. Blackburn, "A3 adenosine receptor signaling contributes to airway mucin secretion after allergen challenge," American Journal of Respiratory Cell and Molecular Biology, vol. 35, no. 5, pp. 549-558, 2006.

[2] M. O. Henke, G. John, M. Germann, H. Lindemann, and B. K. Rubin, "MUC5AC and MUC5B mucins increase in cystic fibrosis airway secretions during pulmonary exacerbation," American Journal of Respiratory and Critical Care Medicine, vol. 175, no. 8, pp. 816-821, 2007.

[3] K. Wang, Y. L. Feng, F. Q. Wen et al., "Increased expression of human calcium-activated chloride channel 1 is correlated with mucus overproduction in the airways of Chinese patients with chronic obstructive pulmonary disease," Chinese Medical Journal, vol. 120, no. 12, pp. 1051-1057, 2007.

[4] M. C. Rose and J. A. Voynow, "Respiratory tract mucin genes and mucin glycoproteins in health and disease," Physiological Reviews, vol. 86, no. 1, pp. 245-278, 2006.

[5] M. R. Knowles and R. C. Boucher, "Mucus clearance as a primary innate defense mechanism for mammalian airways," Journal of Clinical Investigation, vol. 109, no. 5, pp. 571-577, 2002.

[6] M. C. Rose, T. J. Nickola, and J. A. Voynow, "Airway mucus obstruction: mucin glycoproteins, MUC gene regulation and goblet cell hyperplasia," American Journal of Respiratory Cell and Molecular Biology, vol. 25, no. 5, pp. 533-537, 2001.

[7] J. H. Vernooy, M. Küçükaycan, J. A. Jacobs et al., "Local and systemic inflammation in patients with chronic obstructive pulmonary disease: soluble tumor necrosis factor receptors are increased in sputum," American Journal of Respiratory and Critical Care Medicine, vol. 166, no. 9, pp. 1218-1224, 2002.

[8] J. H. Yoon, T. Gray, K. Guzman, J. S. Koo, and P. Nettesheim, "Regulation of the secretory phenotype of human airway epithelium by retinoic acid, triiodothyronine, and extracellular matrix," American Journal of Respiratory Cell and Molecular Biology, vol. 16, no. 6, pp. 724-731, 1997.

[9] T. Gray, P. Nettesheim, C. Basbaum, and J. S. Koo, "Regulation of mucin gene expression in human tracheobronchial epithelial cells by thyroid hormone," Biochemical Journal, vol. 353, no. 3, pp. 727-734, 2001.

[10] T. Gray, J. S. Koo, and P. Nettesheim, "Regulation of mucous differentiation and mucin gene expression in the tracheobronchial epithelium," Toxicology, vol. 160, no. 1-3, pp. 35-46, 2001.

[11] J. S. Koo, J. H. Yoon, T. Gray, D. Norford, A. M. Jetten, and P. Nettesheim, "Restoration of the mucous phenotype by retinoic acid in retinoid-deficient human bronchial cell cultures: changes in mucin gene expression," American Journal of Respiratory Cell and Molecular Biology, vol. 20, no. 1, pp. 4352, 1999.

[12] P. J. Tai, Y. H. Huang, C. H. Shih et al., "Direct regulation of androgen receptor-associated protein 70 by thyroid hormone and its receptors," Endocrinology, vol. 148, no. 7, pp. 34853495, 2007. 
[13] M. Beato, "Transcriptional control by nuclear receptors," FASEB Journal, vol. 5, no. 7, pp. 2044-2051, 1991.

[14] G. Graupner, K. N. Wills, M. Tzukerman, X. Zhang, and M. Pfahl, "Dual regulatory role for thyroid-hormone receptors allows control of retinoic-acid receptor activity," Nature, vol. 340, no. 6235, pp. 653-656, 1989.

[15] M. Tomic, C. K. Jiang, H. S. Epstein, I. M. Freedberg, H. H. Samuels, and M. Blumenberg, "Nuclear receptors for retinoic acid and thyroid hormone regulate transcription of keratin genes," Cell regulation, vol. 1, no. 12, pp. 965-973, 1990.

[16] S. K. Moon, J. H. Yoon, H. N. Kim, D. J. Lim, and M. H. Chung, "Effects of retinoic acid, triiodothyronine and hydrocortisone on mucin and lysozyme expression in cultured human middle ear epithelial cells," Acta Oto-Laryngologica, vol. 120, no. 8, pp. 944-949, 2000.

[17] C. A. Hewson, M. R. Edbrooke, and S. L. Johnston, "PMA induces the MUC5AC respiratory mucin in human bronchial epithelial cells, via PKC, EGF/TGF- $\alpha$, Ras/Raf, MEK, ERK and Sp1-dependent mechanisms," Journal of Molecular Biology, vol. 344, no. 3, pp. 683-695, 2004.

[18] J.M. Weitzel, "To bind or not to bind - how to down-regulate target genes by liganded thyroid hormone receptor?" Thyroid Research, vol. 11, no. 1, p. 4, 2008.

[19] M. A. Lazar, "Thyroid hormone action: a binding contract," Journal of Clinical Investigation, vol. 112, no. 4, pp. 497-499, 2003. 


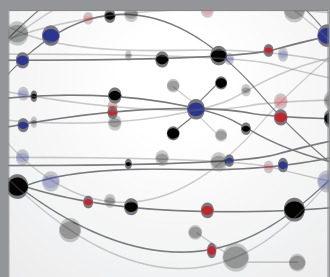

The Scientific World Journal
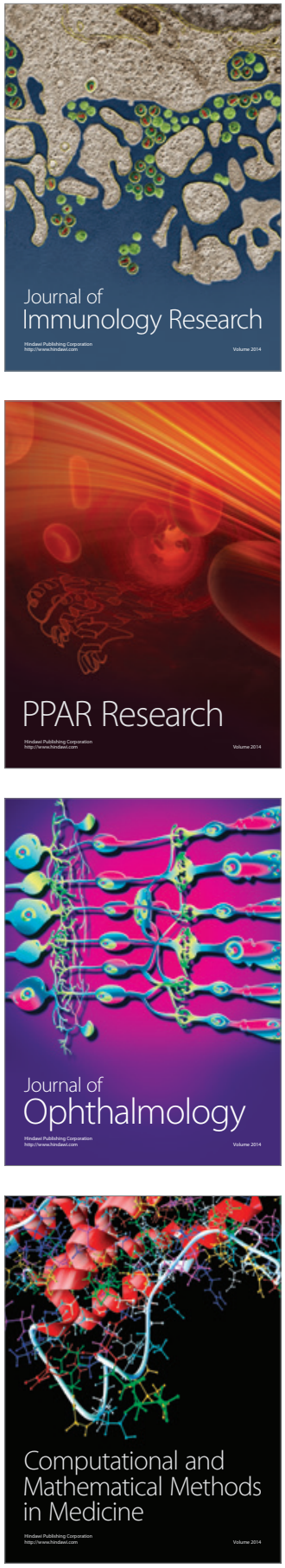

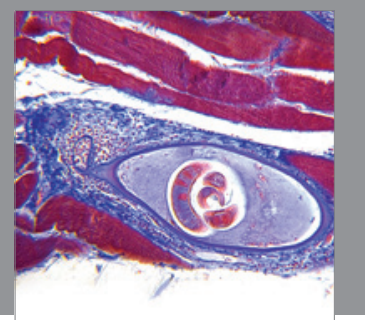

Gastroenterology

Research and Practice
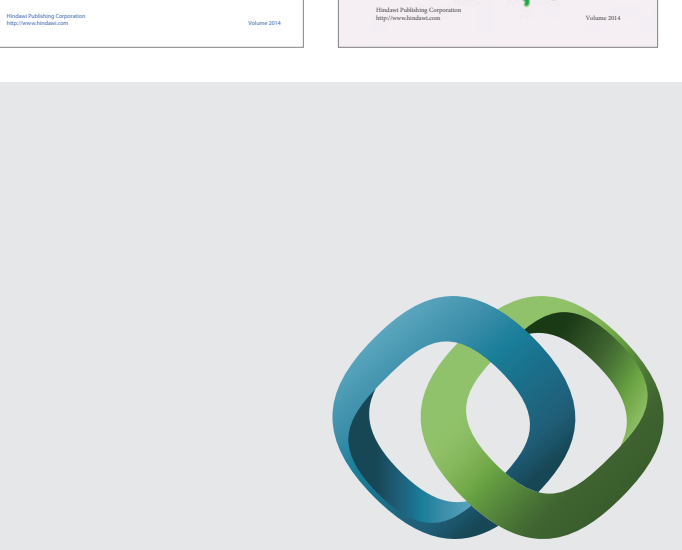

\section{Hindawi}

Submit your manuscripts at

http://www.hindawi.com
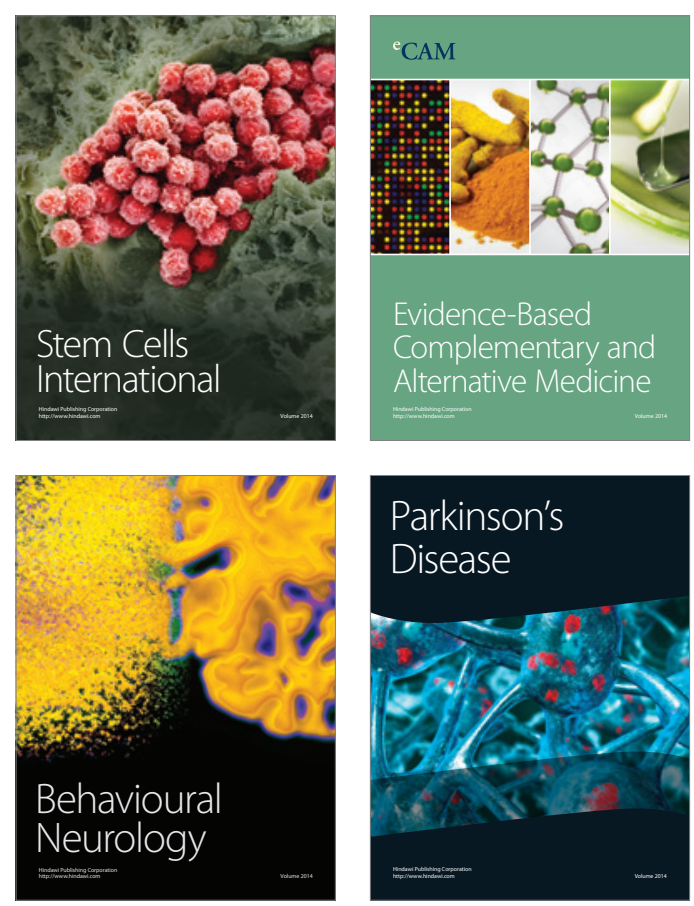

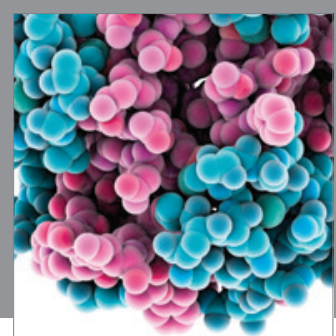

Journal of
Diabetes Research

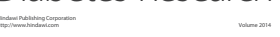

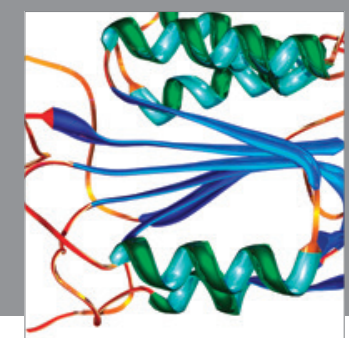

Disease Markers
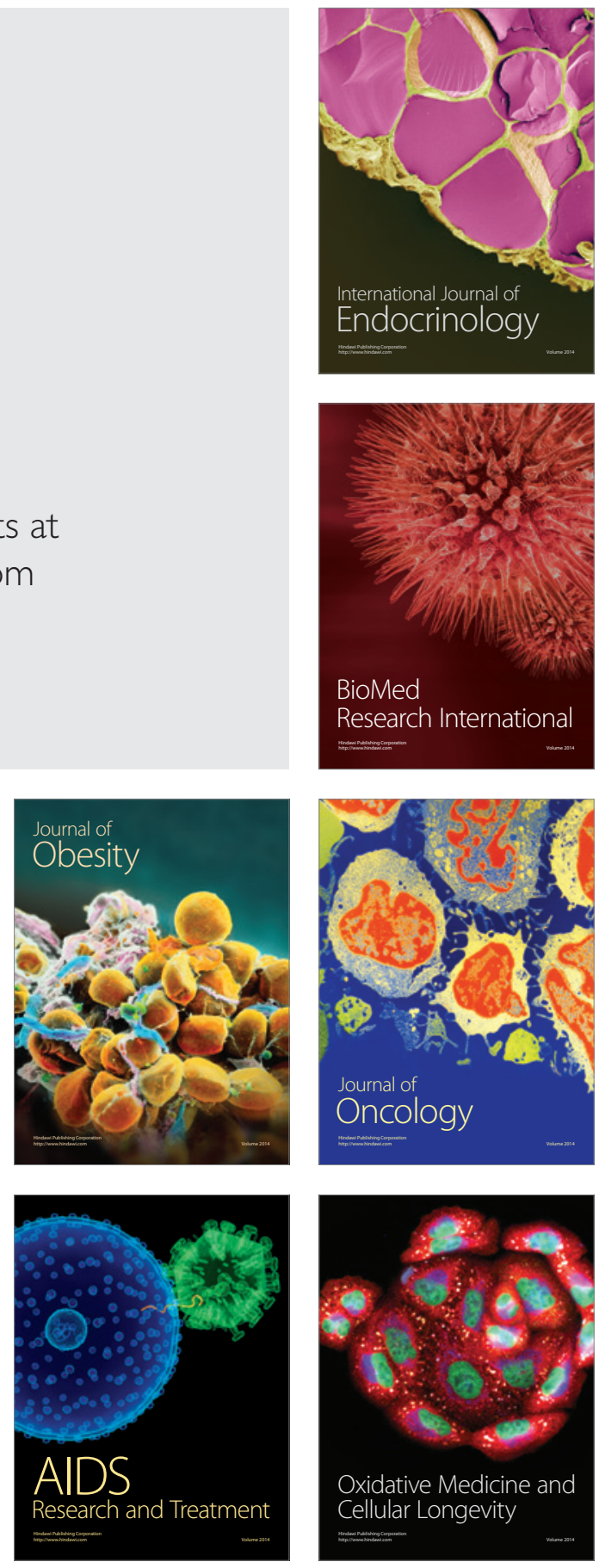